\title{
The complicated effects of obesity on cancer and immunotherapy
}

\author{
Ziming Wang ${ }^{1}$, Arta M Monjazeb² \& William J Murphy*,1,3 \\ ${ }^{1}$ Department of Dermatology, UC Davis School of Medicine, Sacramento, CA 95816, USA \\ ${ }^{2}$ Department of Radiation Oncology, UC Davis Comprehensive Cancer Center, UC School of Medicine, Sacramento, CA 95817, \\ USA \\ ${ }^{3}$ Department of Internal Medicine, Division of Hematology \& Oncology, UC Davis School of Medicine, Sacramento, CA 95817, USA \\ *Author for correspondence: wmjmurphy@ucdavis.edu
}

"Obesity is inherently a complex physiologic process with pleiotropic effects on multiple hormonal and feedback signaling pathways. It will be important to ascertain mechanism of action from an immunological perspective in order to use obesity as a biomarker or prognostic marker in cancer."

First draft submitted: 4 September 2017; Accepted for publication: 5 October 2018; Published online: 7 December 2018

Keywords: cancer $\bullet$ chimeric antigen receptor T cell $\bullet$ inflammaging • immunotherapy • obesity

Immunotherapy has dramatically altered the landscape of cancer treatment with recent successes being reported across multiple advanced cancers. Approaches including immune checkpoint blockade [1-3], chimeric antigen receptor T cell therapy [4] and oncolytic tumor viruses [5] have gained the US FDA approval for various cancers. The importance of immune therapy has been highlighted by the durable responses observed in some patients with metastatic or otherwise resistant tumors. However, despite these successes, serious questions remain in that only a percentage of patients benefit and in some cases life-threatening toxicities can occur. While the vast majority of cancer research and therapies have focused on the cancer itself, immune therapy requires a deep understanding of the patient's immune status as well since this is likely as important as any tumor-related factors with regard to efficacy. Thus, in order to better target and apply immune therapy to patient populations that will respond, it is imperative to understand factors that affect overall immune responses given a genetically heterogeneous, increasingly aged and diverse population. Obvious parameters affecting immune status, outside of genetics center on: age, gender, prior immune exposures/conditions, overall health and comorbidities and more recently, obesity.

Obesity $(\mathrm{BMI} \geq 30)$ is reaching pandemic proportions in the USA [6] with the concerning trend of increasing prevalence in younger age-groups [7]. Obesity is considered a negative prognostic factor for multiple pathologic states, including cancer. The impact of obesity on immune status is still poorly understood, in part given the pleotropic effects of obesity and the multiple confounding factors which accompany obesity. Obesity is commonly associated with what is called a 'metainflammatory' state in which increased pro-inflammatory immune cells (i.e., M1 macrophages, Th1 and $\mathrm{CD} 8^{+} \mathrm{T}$ cells, etc), which permeate every tissue, are geared to release pro-inflammatory cytokines (IL6, TNF) causing chronic inflammation and tissue damage [8]. Further, this inflammatory state is generally thought to be immunosuppressive on all aspects of immune responses and the term 'inflammaging' is coined to describe the impaired adaptive immune response status that occurs $[9,10]$. A problem in studying obesity in cancer-immune interactions is that obesity may directly affect the cancer itself. Obesity has been reported to be a risk factor for both cancer incidence and promoting cancer progression [11-13] with attention being given to the high nutrient rich environment promoting cancer growth and inflammation promoting cancer angiogenesis and invasive properties [14]. Thus, outside of even immune suppression due to inflammaging, obesity is thought to have a negative overall impact on cancer progression and outcome regardless of therapeutic approaches.

Recent clinical studies, however, have seriously called into question this thinking, particularly in the context of immunotherapy. Surprisingly, it was recently reported that improved survival was observed with cancer outcome in men receiving targeted therapy (including immunotherapy with anti-PD1/PDL1) with high BMI [15]. The mech-

Future Medicine 
anisms underlying this differential effect due to BMI on outcome were not clear, much less the gender differences. However, before extrapolating these findings to immunotherapy approaches in general, several caveats need to be always considered. It is important to take into account that, despite the tendency to lump all immunotherapies in one category, immunotherapeutic approaches are not equivalent in both target/application, mechanisms of action and extent of effects (including off-target effects/toxicities) just as not all cancers are to be viewed the same for immunotherapy application. In that regard, preclinical studies may shed further insights as to possible mechanisms of action, both good and bad, influencing outcome/efficacy. Therein reside potential problems as preclinical studies in cancer research center on genetically inbred laboratory mice housed under specific pathogen free (SPF) conditions severely limiting pathogen exposure much less active infections that normally occur in humans. The advantages of these models are the ease: cost and ability to perform mechanistic studies in a timely and reproducible manner. There are significant weaknesses in mouse tumor models, however, that need to always be taken into consideration before extrapolating potential clinical significance. These include the obvious and considerable species differences, the use of laboratory inbred genetically identical mice under SPF conditions [16] limiting immune exposure, the use of models involving the implantation of extensively cultured tumor cell lines into healthy mice as a 'cancer model' for therapy, and the prevalent use of young mice (the age equivalent of human adolescent) [17]. Indeed, simply generating diet-induced obese mice takes time, usually 3-4 months, which automatically changes the baseline immune status of age-matched mice on control diets versus the young mice (8-12 weeks of age) more commonly used. Additionally, the types of diets used to induce obesity include different percentages of fat with or without added sugars (the so-called 'western diet') all of which have significant impact on nonimmune parameters depending on extent and duration. This is in contrast to obese mice resulting from genetic disruption of the leptin pathway, which impacts food intake and these mice become rapidly obese on regular diets. All of these variables, therefore, need to be considered when studying obesity in preclinical models given the profound metabolic and immune differences that result from these different models.

We have reported that immunotherapy consisting of strong systemic stimulation (i.e., anti-CD 40 and high dose IL2, $\alpha$ CD40/IL-2) administered to young healthy tumor-bearing mice results in significant antitumor effects, whereas the exact same regimen given to older mice resulted in rapid lethality $[18,19]$. One mechanism underlying these opposing effects was the increased in adiposity observed in laboratory mice as they age [18]. Obese mice, even when using younger mice, also demonstrated increased immunotherapy associated toxicities in part mediated by 'cytokine storm' due to macrophages. Recently, however, we observed that, in contrast to the effects of systemic immunostimulatory regimens, use of checkpoint blockade had the opposite outcome. Obese mice displayed greater antitumor effects and survival compared with lean control mice. Analysis of clinical outcomes in patients treated with checkpoint blockade immunotherapy corroborated these findings. Therapy resulted in increased antitumor effects by checkpoint blockade despite the fact that the tumors grew faster in obese recipients and the recipients showed evidence of greater immunosuppression compared with controls (Figure 1). Thus, despite higher 'inflammaging' in obese mice and increased PD1/PDL1 expression associated with decreased T cell function, the application of anti-PD1 ( $\alpha \mathrm{PD}-1)$ resulted in greater antitumor effects. Importantly, no increases in adverse effects/toxicities were noted using this approach in obese subjects which in contrast to the effects of immunostimulatory regimens such as $\alpha \mathrm{CD} 40 / \mathrm{IL}-2$ [20]. These results highlight several important conclusions. First and foremost, human modifying conditions/variables outside of genetics, such as aging and obesity, need to be taken into consideration with preclinical assessments. We observed profound differences between lean and obese mice treated with the same immunotherapy resulting in contrasting outcomes despite the fact that they were genetically identical. Second, the type/manner of immunotherapy applied needs to be considered as completely different outcomes (death from cytokine storm and toxicities vs increased survival with no toxicities) could be observed comparing immunostimulatory high dose IL2 versus $\alpha$ PD-1 treatment in obese mice. Finally, confounding factors such as aging in conjunction with obesity can further distort the impact (either positive or negative) on immunotherapy efficacy as both immune and nonimmune cell-types are impacted. Finally, species-specific differences (i.e., circadian rhythm, social status and genetic differences between inbred stains) and laboratory mouse husbandry practices all impact immune responses, particularly in context of SPF facilities. Clearly, there is much more work that needs to be done in dissecting the impact of obesity on cancer immunotherapy. What are the mechanisms underlying 'inflammaging' and how does one best mirror the clinical scenario using laboratory mice? Does the duration of obesity impact extent of exhaustion? Does the diet itself impact differentially independent of body fat deposition? Finally, how does gender affect outcome given reports that only high BMI males demonstrated this beneficial effect [15]? Obesity is inherently a complex physiologic process with pleiotropic effects on multiple hormonal 


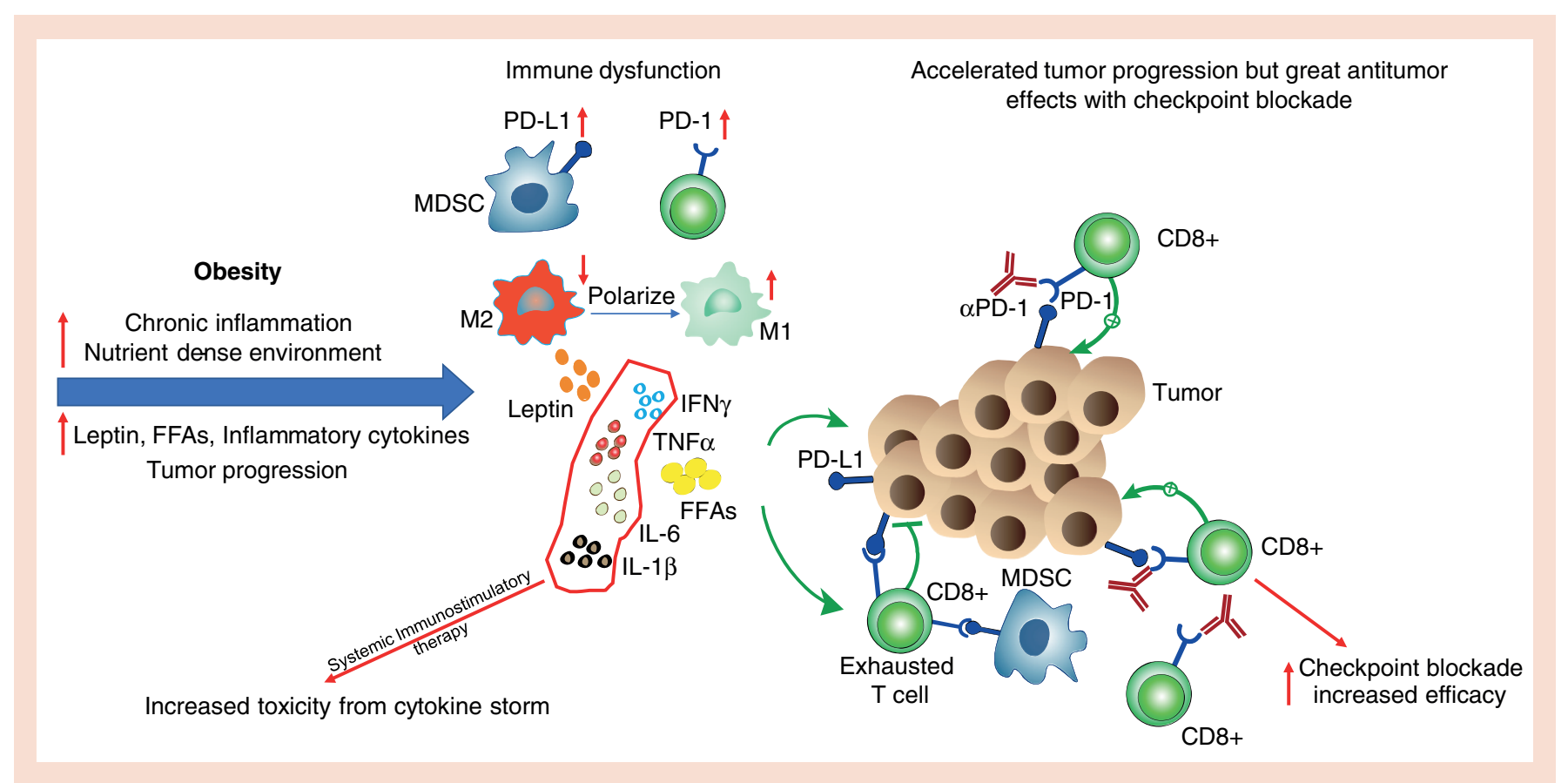

Figure 1. The complicated effects of obesity on cancer and immunotherapy. The nutrient-dense environment of obesity causes chronic inflammation, associated with increased levels of leptin, free fatty acids (FFAs) and inflammatory cytokines (IFN $\gamma$, TNF $\alpha$, IL-6, IL-1 $\beta$, etc.), which upregulate the expression of PD-1 and PD-L1 on tumor-infiltrating cytotoxic CD8 ${ }^{+} \mathrm{T}$ cells and myeloid-derived suppressive cells (MDSCs), respectively. The inflammatory obese environment polarizes anti-inflammatory M2 macrophages to inflammatory M1 macrophages. Systemic immunostimulatory therapy (i.e., $\alpha$ CD40/IL-2) leads to fatal toxicity from cytokine storm. The complicated immune dysfunction caused by obesity leads to T-cell exhaustion and accelerated tumor progression. However, $\alpha$ PD-1 monotherapy results in amplified antitumor effects within the inflammatory environment of obesity.

and feedback signaling pathways. It will be important to ascertain mechanism of action from an immunological perspective in order to use obesity as a biomarker or prognostic marker in cancer. Thus, current paradigms are being altered in which obesity, while associated with numerous negative indicators in cancer, can be exploited for positive outcomes if affected pathways are properly targeted.

Financial \& competing interest disclosure

The authors have no relevant affiliations or financial involvement with any organization or entity with a financial interest in or financial conflict with the subject matter or materials discussed in the manuscript. This includes employment, consultancies, honoraria, stock ownership or options, expert testimony, grants or patents received or pending, or royalties.

No writing assistance was utilized in the production of this manuscript.

\section{References}

1 Larkin J et al. Combined nivolumab and ipilimumab or monotherapy in untreated melanoma. N. Engl. J. Med. 373, 23-34 (2015).

2 Garon EB et al. Pembrolizumab for the treatment of non-small-cell lung cancer. N. Engl. J. Med. 372, 2018-2028 (2015).

3 Powles T et al. MPDL3280A (anti-PD-L1) treatment leads to clinical activity in metastatic bladder cancer. Nature 515, 558-562, doi:10.1038/nature13904 (2014) (Epub ahead of print).

4 June CH, O’Connor RS, Kawalekar OU, Ghassemi S, Milone MC. CAR T cell immunotherapy for human cancer. Science 359, 1361-1365 (2018).

5 Kaufman HL, Kohlhapp FJ, Zloza A. Oncolytic viruses: a new class of immunotherapy drugs. Nat. Rev. Drug Discov. 14, 642 (2015).

6 Cawley J, Meyerhoefer C. The medical care costs of obesity: an instrumental variables approach. J. Health Econ. 31, 219-230 (2012).

7 Hales CM, Carroll MD, Fryar CD, Ogden CL. Prevalence of obesity among adults and youth: United States, 2015-2016. NCHS Data Brief2017. www.cdc.gov/nchs/data/databriefs/db288.pdf

8 Castoldi A, Naffah de Souza C, Câmara NOS, Moraes-Vieira PM. The macrophage switch in obesity development. Front. Immunol. 6, 637 (2016). 
9 Frasca D, Blomberg BB. Inflammaging decreases adaptive and innate immune responses in mice and humans. Biogerontology 17, 7-19 (2016).

10 Decman $\mathrm{V}$ et al. Defective CD8 $\mathrm{T}$ cell responses in aged mice are due to quantitative and qualitative changes in virus-specific precursors. J.Immunol. 1101098 188(4), 1933-1941 (2012).

11 Renehan AG, Tyson M, Egger M, Heller RF, Zwahlen M. Body-mass index and incidence of cancer: a systematic review and meta-analysis of prospective observational studies. Lancet 371, 569-578 (2008).

12 Calle EE, Rodriguez C, Walker-Thurmond K, Thun MJ. Overweight, obesity, and mortality from cancer in a prospectively studied cohort of US adults. N. Engl. J. Med. 348, 1625-1638 (2003).

13 Arnold M et al. Global burden of cancer attributable to high body-mass index in 2012: a population-based study. Lancet Oncol. 16, 36-46 (2015).

14 Deng T, Lyon CJ, Bergin S, Caligiuri MA, Hsueh WA. Obesity, Inflammation, and Cancer. Annu. Rev. Pathol. 11, 421-449, doi:10.1146/annurev-pathol-012615-044359 (2016) (Epub ahead of print).

15 McQuade JL et al. Association of body-mass index and outcomes in patients with metastatic melanoma treated with targeted therapy, immunotherapy, or chemotherapy: a retrospective, multicohort analysis. Lancet Oncol. 19(3), 310-322 (2018).

16 Beura LK et al. Normalizing the environment recapitulates adult human immune traits in laboratory mice. Nature 532, 512 (2016).

17 Rangarajan A, Weinberg RA. Comparative biology of mouse versus human cells: modelling human cancer in mice. Nat. Rev. Cancer 3 , $952(2003)$.

18 Mirsoian A et al. Adiposity induces lethal cytokine storm after systemic administration of stimulatory immunotherapy regimens in aged mice. J. Exp. Med. 211, 2373-2383, doi:10.1084/jem.20140116 (2014) (Epub ahead of print).

19 Bouchlaka MN, Murphy WJ. Impact of aging in cancer immunotherapy: the importance of using accurate preclinical models. Oncoimmunology2, e27186 (2013).

20 Wang $\mathrm{Z}$ et al. Paradoxical effects of obesity on T cell function during tumor progression and PD-1 checkpoint blockade. Nat.Med. (12 November 2018). doi: 10.1038/s41591-018-0221-5. (Epub ahead of print). 